ANNALES

POLONICI MATHEMATICI

LXXV.3 (2000)

\title{
A viscoelastic contact problem with normal damped response and friction
}

by B. Awbi (Perpignan), El H. Essoufi (Clermont-Ferrand) and

\author{
M. Sofonea (Perpignan)
}

\begin{abstract}
We study an evolution problem which describes the quasistatic contact of a viscoelastic body with a foundation. We model the contact with normal damped response and a local friction law. We derive a variational formulation of the model and we establish the existence of a unique weak solution to the problem. The proof is based on monotone operators and fixed point arguments. We also establish the continuous dependence of the solution on the contact boundary conditions.
\end{abstract}

1. Introduction. Frictional contact between deformable bodies can be frequently found in industry and everyday life. Because of the importance in metal forming and automotive industry, a considerable effort has been made with the modeling and numerical simulations of contact problems, and the engineering literature concerning this topic is rather extensive.

An early attempt to study contact problems for linear elastic and viscoelastic materials within the framework of variational inequalities was made in [5]. An excellent reference on the field of contact problems with or without friction is [7]. The mathematical, mechanical and numerical state of the art can be found in the proceedings [11]. The quasistatic approximation of contact problems is obtained when the applied forces in the system vary slowly with time and therefore the inertial terms in the equation of motion can be neglected. There has been considerable interest in the study of quasistatic contact problems recently (see, e.g., $[1,2,4,8,12,13]$ and the references therein).

Quasistatic contact problems for viscoelastic materials with a general damped response condition and friction have been studied recently in [14]. There the friction has been modeled with a version of Coulomb's law and

2000 Mathematics Subject Classification: 73F15, 73V25, 49J40, 47H15.

Key words and phrases: viscoelastic material, frictional contact, normal damped response, variational problem, monotone operator, fixed point. 
therefore the model was set as a family of variational inequalities for the velocity field, parametrized with time. The existence of a unique weak solution of the model has been proved using results on elliptic variational inequalities and fixed point arguments.

In this paper we investigate the process of quasistatic frictional contact between a deformable body and a foundation. The body is assumed to be viscoelastic with a nonlinear constitutive law. The contact is modeled with normal damped response, which represents the possible behavior of a layer lubricant on the contact surface. Both the viscoelastic constitutive law and the damped response condition are chosen as in [14]. We model the friction assuming that the tangential shear on the contact surface is given as a function of the tangential velocity. Considering friction laws of this form leads to a new mathematical model involving a family of nonlinear evolution equations parametrized with time. We establish the existence of a unique solution of the model using again fixed point theorems, but for different operators, since the contact conditions, and therefore the settings, in [14] and here are different. Then we prove the stability of the problem with respect to perturbations of the contact conditions, which is important from the point of view of applications.

The paper is organized as follows. In Section 2 we introduce some notation and preliminary material. In Section 3 we present the mechanical problem, derive its variational formulation and state our main existence and uniqueness result, Theorem 3.1. The proof of Theorem 3.1 is given in Section 4. Finally, in Section 5 we study the dependence of the solution on the contact boundary conditions and we establish a convergence result, Theorem 5.1.

2. Notation and preliminaries. In this short section, we present the notation we shall use and some preliminary material. For further details, we refer the reader to $[5,6,10]$.

We denote by $S_{N}$ the space of second order symmetric tensors on $\mathbb{R}^{N}$ $(N=2,3)$, while ". " and $|\cdot|$ will represent the inner product and the Euclidean norm on $S_{N}$ and $\mathbb{R}^{N}$, respectively. Let $\Omega \subset \mathbb{R}^{N}$ be a bounded domain with a Lipschitz boundary $\Gamma$ and let $\nu$ denote the unit outer normal on $\Gamma$. In what follows the indices $i$ and $j$ run between 1 and $N$, the summation convention over repeated indices is adopted and the index that follows a comma indicates a partial derivative with respect to the corresponding component of the independent variable. We denote by $D(\Omega)$ the space of infinitely differentiable real-valued functions with compact support in $\Omega$ and by $L^{2}(\Omega)$ the space of measurable and square summable real-valued functions on $\Omega$. We use the notation $H^{1 / 2}(\Gamma)$ for the Sobolev space of order $s=1 / 2$ on $\Gamma$ (see, e.g., [5], p. 42) while $\mathcal{D}$ and $H_{\Gamma}$ denote the spaces 


$$
\mathcal{D}=\left\{\varphi=\left(\varphi_{i}\right) \mid \varphi_{i} \in D(\Omega)\right\}, \quad H_{\Gamma}=\left\{\theta=\left(\theta_{i}\right) \mid \theta_{i} \in H^{1 / 2}(\Gamma)\right\} .
$$

Throughout the paper we also use the following notation:

$$
\begin{aligned}
H & =\left\{u=\left(u_{i}\right) \mid u_{i} \in L^{2}(\Omega)\right\}, \quad \mathcal{H}=\left\{\sigma=\left(\sigma_{i j}\right) \mid \sigma_{i j}=\sigma_{j i} \in L^{2}(\Omega)\right\}, \\
H_{1} & =\left\{u=\left(u_{i}\right) \mid \varepsilon(u) \in \mathcal{H}\right\}, \quad \mathcal{H}_{1}=\{\sigma \in \mathcal{H} \mid \operatorname{Div} \sigma \in H\} .
\end{aligned}
$$

Here $\varepsilon$ and Div denote the deformation and the divergence operators, respectively, defined by

$$
\varepsilon(u)=\left(\varepsilon_{i j}(u)\right), \quad \varepsilon_{i j}(u)=\frac{1}{2}\left(u_{i, j}+u_{j, i}\right), \quad \operatorname{Div} \sigma=\left(\sigma_{i j, j}\right) .
$$

The spaces $H, \mathcal{H}, H_{1}$ and $\mathcal{H}_{1}$ are real Hilbert spaces endowed with their canonical inner products given by

$$
\begin{array}{cc}
\langle u, v\rangle_{H}=\int_{\Omega} u_{i} v_{i} d x, \quad\langle\sigma, \tau\rangle_{\mathcal{H}}=\int_{\Omega} \sigma_{i j} \tau_{i j} d x, \\
\langle u, v\rangle_{H_{1}}=\langle u, v\rangle_{H}+\langle\varepsilon(u), \varepsilon(v)\rangle_{\mathcal{H}}, \quad\langle\sigma, \tau\rangle_{\mathcal{H}_{1}}=\langle\sigma, \tau\rangle_{\mathcal{H}}+\langle\operatorname{Div} \sigma, \operatorname{Div} \tau\rangle_{H} .
\end{array}
$$

The associated norms are denoted by $|\cdot|_{H},|\cdot|_{\mathcal{H}},|\cdot|_{H_{1}}$ and $|\cdot|_{\mathcal{H}_{1}}$, respectively.

Let $\gamma: H_{1} \rightarrow H_{\Gamma}$ be the trace map. For every $u \in H_{1}$, we still write $u$ for the trace $\gamma u$ of $u$ on $\Gamma$ and we denote by $u_{\nu}$ and $u_{\tau}$ the normal and tangential components of $u$ on $\Gamma$ given by

$$
u_{\nu}=u \cdot \nu, \quad u_{\tau}=u-u_{\nu} \nu .
$$

Let $H_{\Gamma}^{\prime}$ be the dual of $H_{\Gamma}$ and let $\langle\cdot, \cdot\rangle$ denote the duality pairing between $H_{\Gamma}^{\prime}$ and $H_{\Gamma}$. For every $\sigma \in \mathcal{H}_{1}, \sigma \nu$ can be defined as the element in $H_{\Gamma}^{\prime}$ which satisfies

$$
\langle\sigma \nu, \gamma u\rangle=\langle\sigma, \varepsilon(u)\rangle_{\mathcal{H}}+\langle\operatorname{Div} \sigma, u\rangle_{H} \quad \forall u \in H_{1} .
$$

Let also $\sigma_{\nu}$ and $\sigma_{\tau}$ represent the normal and tangential traces of $\sigma$, respectively. If $\sigma$ is a smooth function, e.g. $\sigma \in C^{1}$, then

$$
\langle\sigma \nu, \gamma u\rangle=\int_{\Gamma} \sigma \nu \cdot u d a \quad \forall u \in H_{1}
$$

where $d a$ is the surface measure element, and

$$
\sigma_{\nu}=(\sigma \nu) \cdot \nu, \quad \sigma_{\tau}=\sigma \nu-\sigma_{\nu} \nu
$$

Finally, let $\left(X,|\cdot|_{X}\right)$ be a real normed space. Then $C(0, T ; X)$ and $C^{1}(0, T ; X)$ denote the spaces of continuous and continuously differentiable functions from $[0, T]$ to $X$, with the norms

$$
|x|_{C(0, T ; X)}=\max _{t \in[0, T]}|x(t)|_{X}, \quad|x|_{C^{1}(0, T ; X)}=\max _{t \in[0, T]}\left(|x(t)|_{X}+|\dot{x}(t)|_{X}\right),
$$

respectively. Here and everywhere below, the dot above a symbol represents the time derivative. 
3. Problem statement and variational formulation. In this section we describe the model for the process, present its variational formulation and state our main existence and uniqueness result, Theorem 3.1.

The physical setting is as follows. We consider a deformable body which occupies a domain $\Omega$ and let $\Gamma_{1}, \Gamma_{2}, \Gamma_{3}$ be a partition of $\Gamma$ into three disjoint measurable parts such that meas $\Gamma_{1}>0$. Let $T>0$ and let $[0, T]$ denote the time interval considered. We assume that the body is fixed on $\Gamma_{1} \times(0, T)$ and therefore the displacement field vanishes there. Surface tractions of density $f_{2}$ act on $\Gamma_{2} \times(0, T)$ and a volume force of density $f_{0}$ acts on $\Omega \times(0, T)$. Moreover, the solid is in frictional contact with a foundation on $\Gamma_{3} \times(0, T)$.

We denote by $u$ the displacement field, by $\sigma$ the stress field, and $\varepsilon(u)$ represents the small strain tensor. We assume that the material is viscoelastic and has a general constitutive law of the form

$$
\sigma=\mathcal{A}(\varepsilon(\dot{u}))+G(\varepsilon(u))
$$

where $\mathcal{A}$ and $G$ are given nonlinear constitutive functions. We recall that in linear viscoelasticity, the stress tensor $\sigma=\left(\sigma_{i j}\right)$ is given by

$$
\sigma_{i j}=a_{i j k l} \varepsilon_{k l}(\dot{u})+g_{i j k l} \varepsilon_{k l}(u)
$$

where $\mathcal{A}=\left(a_{i j k l}\right)$ is the viscosity tensor and $G=\left(g_{i j k l}\right)$ is the elasticity tensor, for $i, j, k, l=1, \ldots, N$. Kelvin-Voigt viscoelastic materials of the form (3.1) involving nonlinear constitutive functions have been considered recently in [12-14].

We now turn to describing the contact conditions, which are our main object of interest. We assume that the normal stress $\sigma_{\nu}$ satisfies a general normal damped response condition

$$
-\sigma_{\nu}=p_{\nu}\left(\dot{u}_{\nu}\right)
$$

where $\dot{u}_{\nu}$ represents the normal velocity and $p_{\nu}$ is a prescribed function. Equality (3.2) states a general dependence of the normal stress on the normal velocity. In the case when

$$
p_{\nu}(r)=k r
$$

with $k \geq 0$, the resistance of the foundation to penetration is proportional to the normal velocity. This type of behavior was considered in [15] when modeling the motion of a deformable body on sand or a granular material. We may also consider the case

$$
p_{\nu}(r)=\beta\left(r_{+}\right)^{m}+p_{0}
$$

where $r_{+}=\max \{0, r\}$ and $0<m \leq 1$. For $m=1$ the boundary condition (3.4) was considered in [13] where the potential contact surface $\Gamma_{3}$ was assumed to be covered with a lubricant that contains solid particles, such as 
one of the new smart lubricants, or with worn metallic particles. In (3.4), $\beta$ represents the damping resistance constant, assumed positive, and $p_{0}$ is the oil pressure, which is given and nonnegative. The contact conditions (3.2) and (3.4) model the fact that the oil layer presents resistance or damping only when the surface moves towards the foundation.

The associated friction law is chosen as

$$
-\sigma_{\tau}=p_{\tau}\left(\dot{u}_{\tau}\right) .
$$

Here $p_{\tau}$ is a prescribed vector-valued function, $\dot{u}_{\tau}$ denotes the tangential velocity and $\sigma_{\tau}$ represents the tangential force on the contact boundary. As an example we may consider the function

$$
p_{\tau}(r)=\mu|r|^{m-1} r
$$

where $\mu$ represents the coefficient of friction, assumed positive, and $0<$ $m \leq 1$. This is the case when the contact surface is lubricated with a thin layer of non-Newtonian fluid (see e.g. [15]). In the particular case $m=1$, (3.5), (3.6) show that the tangential shear is proportional to the tangential velocity.

With these assumptions, denoting by $u_{0}$ the initial displacement field, the mechanical problem of frictional contact of the viscoelastic body may be formulated classically as follows.

Problem $P$. Find a displacement field $u: \Omega \times[0, T] \rightarrow \mathbb{R}^{N}$ and a stress field $\sigma: \Omega \times[0, T] \rightarrow S_{N}$ such that

$$
\begin{aligned}
& \sigma=\mathcal{A}(\varepsilon(\dot{u}))+G(\varepsilon(u)) \quad \text { in } \Omega \times(0, T), \\
& \operatorname{Div} \sigma+f_{0}=0 \quad \text { in } \Omega \times(0, T) \text {, } \\
& u=0 \quad \text { on } \Gamma_{1} \times(0, T), \\
& \sigma \nu=f_{2} \quad \text { on } \Gamma_{2} \times(0, T) \text {, } \\
& -\sigma_{\nu}=p_{\nu}\left(\dot{u}_{\nu}\right), \quad-\sigma_{\tau}=p_{\tau}\left(\dot{u}_{\tau}\right) \quad \text { on } \Gamma_{3} \times(0, T) \text {, } \\
& u(0)=u_{0} \quad \text { in } \Omega \text {. }
\end{aligned}
$$

To provide the variational formulation of the mechanical problem (3.7)(3.12) we need additional notation. Thus, let $V$ denote the closed subspace of $H_{1}$ defined by

$$
V=\left\{v \in H_{1} \mid v=0 \text { on } \Gamma_{1}\right\} .
$$

Since meas $\Gamma_{1}>0$, the following Korn-type inequality holds: there exists $C_{K}>0$ which depends only on $\Omega$ and $\Gamma_{1}$ such that

$$
|\varepsilon(v)|_{\mathcal{H}} \geq C_{K}|v|_{H_{1}} \quad \forall v \in V .
$$

The proof of this inequality may be found in [9], p. 79. On $V$ we consider 
the inner product given by

$$
\langle u, v\rangle_{V}=\langle\varepsilon(u), \varepsilon(v)\rangle_{\mathcal{H}} \quad \forall u, v \in V,
$$

and let $|\cdot|_{V}$ be the associated norm, i.e.

$$
|v|_{V}=|\varepsilon(v)|_{\mathcal{H}} \quad \forall v \in V .
$$

It follows that $|\cdot|_{H_{1}}$ and $|\cdot|_{V}$ are equivalent norms on $V$ and therefore $\left(V,|\cdot|_{V}\right)$ is a real Hilbert space. Moreover, by the Sobolev trace theorem and (3.13), we have a constant $C_{0}>0$ depending only on $\Omega, \Gamma_{1}$, and $\Gamma_{3}$ such that

$$
|v|_{L^{2}\left(\Gamma_{3}\right)^{N}} \leq C_{0}|v|_{V} \quad \forall v \in V .
$$

In the study of the mechanical problem (3.7)-(3.12), we assume that the viscosity operator $\mathcal{A}: \Omega \times S_{N} \rightarrow S_{N}$ satisfies:

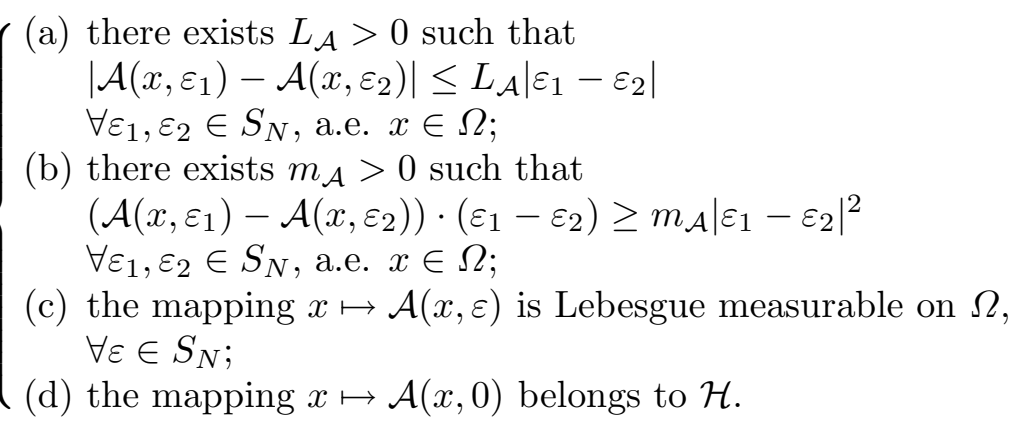

The elasticity operator $G: \Omega \times S_{N} \rightarrow S_{N}$ satisfies:

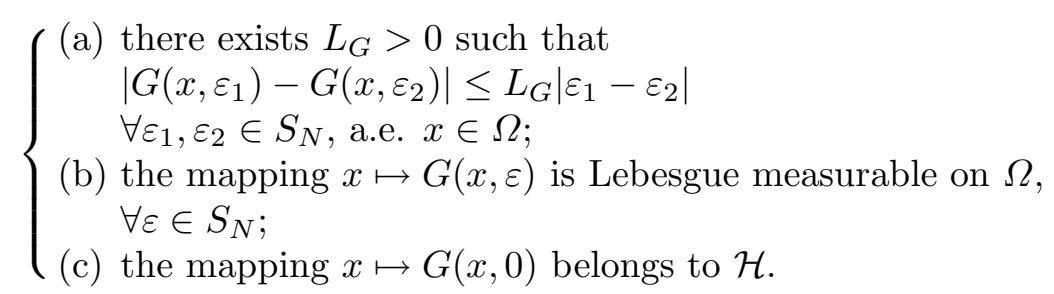

We also assume that the contact functions $p_{\nu}: \Gamma_{3} \times \mathbb{R} \rightarrow \mathbb{R}$ and $p_{\tau}$ : $\Gamma_{3} \times \mathbb{R}^{N} \rightarrow \mathbb{R}^{N}$ satisfy:
(a) there exist $C_{1}^{\nu}, C_{2}^{\nu}>0$ such that $\left|p_{\nu}(x, r)\right| \leq C_{1}^{\nu}|r|+C_{2}^{\nu} \quad \forall r \in \mathbb{R}$, a.e. $x \in \Gamma_{3} ;$
(b) $\left(p_{\nu}\left(x, r_{1}\right)-p_{\nu}\left(x, r_{2}\right)\right)\left(r_{1}-r_{2}\right) \geq 0$ $\forall r_{1}, r_{2} \in \mathbb{R}$, a.e. $x \in \Gamma_{3}$;
(c) the mapping $x \mapsto p_{\nu}(x, r)$ is Lebesgue measurable on $\Gamma_{3}$, $\forall r \in \mathbb{R}$
(d) the mapping $r \mapsto p_{\nu}(x, r)$ is continuous on $\mathbb{R}$, a.e. $x \in \Gamma_{3}$. 


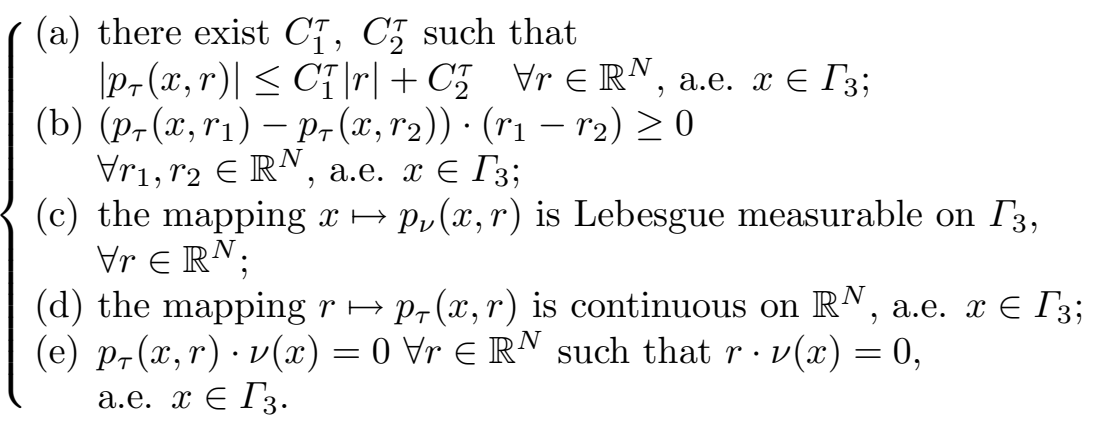

We observe that the assumption (3.19) is satisfied by the functions $p_{\nu}$ defined in (3.3), (3.4) and the assumption (3.20) is satisfied by the function $p_{\tau}$ defined in (3.6). We conclude that our results below are valid for the boundary value problems related to each of these examples.

We suppose that the forces and the tractions have the regularity

$$
f_{0} \in C(0, T ; H), \quad f_{2} \in C\left(0, T ; L^{2}\left(\Gamma_{2}\right)^{N}\right),
$$

and, finally, the initial displacement satisfies

$$
u_{0} \in V \text {. }
$$

Next, we denote by $f(t)$ the element of $V$ given by

$$
\langle f(t), v\rangle_{V}=\left\langle f_{0}(t), v\right\rangle_{H}+\left\langle f_{2}(t), \gamma v\right\rangle_{L^{2}\left(\Gamma_{2}\right)^{N}},
$$

for all $v \in V$ and $t \in[0, T]$, and we note that conditions (3.21) imply

$$
f \in C(0, T ; V) \text {. }
$$

Let $j: V \times V \rightarrow \mathbb{R}$ be the friction functional

$$
j(u, v)=\int_{\Gamma_{3}} p_{\nu}\left(u_{\nu}\right) v_{\nu} d a+\int_{\Gamma_{3}} p_{\tau}\left(u_{\tau}\right) \cdot v_{\tau} d a \quad \forall u, v \in V .
$$

Keeping in mind (3.19) and (3.20) we observe that the integrals in (3.25) are well defined.

Next, we assume that $\{u, \sigma\}$ are regular functions satisfying (3.8)-(3.11) and let $v \in V, t \in[0, T]$. Using (2.2), (2.3) and (3.8) we have

$$
\langle\sigma(t), \varepsilon(v)\rangle_{\mathcal{H}}=\left\langle f_{0}(t), v\right\rangle_{H}+\int_{\Gamma} \sigma(t) \nu \cdot v d a
$$

and, by (3.10) and (3.23), we find

$$
\langle\sigma(t), \varepsilon(v)\rangle_{\mathcal{H}}=\langle f(t), v\rangle_{V}+\int_{\Gamma_{3}} \sigma(t) \nu \cdot v d a
$$

It now follows from (2.1), (2.4) and (3.11) that

$$
\sigma(t) \nu \cdot v=-p_{\nu}\left(\dot{u}_{\nu}(t)\right) v_{\nu}-p_{\tau}\left(\dot{u}_{\tau}(t)\right) \cdot v_{\tau} \quad \text { on } \Gamma_{3}
$$

and therefore from (3.26) and (3.25) we find 


$$
\langle\sigma(t), \varepsilon(v)\rangle_{\mathcal{H}}+j(\dot{u}(t), v)=\langle f(t), v\rangle_{V} .
$$

To conclude, from (3.7), (3.12) and (3.27) we obtain the following variational formulation of the mechanical problem $P$.

Problem $P_{V}$. Find a displacement field $u:[0, T] \rightarrow V$ and a stress field $\sigma:[0, T] \rightarrow \mathcal{H}_{1}$ such that, for $t \in[0, T]$

$$
\begin{gathered}
\sigma(t)=\mathcal{A}(\varepsilon(\dot{u}(t)))+G(\varepsilon(u(t))), \\
\langle\sigma(t), \varepsilon(v)\rangle_{\mathcal{H}}+j(\dot{u}(t), v)=\langle f(t), v\rangle_{V} \quad \forall v \in V, \\
u(0)=u_{0} .
\end{gathered}
$$

The well posedness of the problem $P_{V}$ results from the following existence and uniqueness result, which we establish in the next section.

Theorem 3.1. Assume that (3.17)-(3.22) hold. Then there is a unique solution $\{u, \sigma\}$ to the problem (3.28)-(3.30). Moreover, the solution satisfies

$$
u \in C^{1}(0, T ; V), \quad \sigma \in C\left(0, T ; \mathcal{H}_{1}\right) .
$$

Let $\{u, \sigma\}$ be the solution specified in Theorem 3.1. Then, using standard arguments (see, e.g., $[5,10])$ it follows that

$$
\operatorname{Div} \sigma(t)+f_{0}(t)=0 \quad \text { a.e. in } \Omega,
$$

for all $t \in[0, T]$. Moreover, the following equalities hold, in the sense of traces, for all $t \in[0, T]$ :

$$
\begin{gathered}
u(t)=0 \quad \text { a.e. on } \Gamma_{1}, \\
\sigma(t) \nu=f_{2}(t) \quad \text { a.e. on } \Gamma_{2}, \\
-\sigma_{\nu}(t)=p_{\nu}\left(\dot{u}_{\nu}(t)\right), \quad-\sigma_{\tau}(t)=p_{\tau}\left(\dot{u}_{\tau}(t)\right) \quad \text { a.e. on } \Gamma_{3} .
\end{gathered}
$$

Keeping in mind (3.28), (3.30), (3.32)-(3.35), we may consider $\{u, \sigma\}$ as the weak solution of the mechanical problem $P$. Therefore, by Theorem 3.1 we conclude that under the assumptions (3.17)-(3.22), problem $P$ has a unique weak solution with the regularity (3.31).

4. Proof of Theorem 3.1. The proof of Theorem 3.1 will be carried out in several steps. It is based on fixed point arguments, similar to those used in [12-14] but with a different choice of the operators. We assume in what follows that (3.17)-(3.22) hold. We start with the following existence and uniqueness result.

Lemma 4.1. For every $\eta \in C(0, T ; \mathcal{H})$, there exists a unique couple $\left\{v_{\eta}, \sigma_{\eta}\right\}$ of functions such that, for $t \in[0, T]$,

$$
\begin{gathered}
\sigma_{\eta}(t)=\mathcal{A}\left(\varepsilon\left(v_{\eta}(t)\right)\right)+\eta(t), \\
\left\langle\sigma_{\eta}(t), \varepsilon(v)\right\rangle_{\mathcal{H}}+j\left(v_{\eta}(t), v\right)=\langle f(t), v\rangle_{V} \quad \forall v \in V .
\end{gathered}
$$

Moreover, $v_{\eta} \in C(0, T ; V)$ and $\sigma_{\eta} \in C\left(0, T ; \mathcal{H}_{1}\right)$. 
Proof. Using Riesz's representation theorem we define the operator $B: V \rightarrow V$ and the element $f_{\eta}(t) \in V$ by

$$
\begin{aligned}
\langle B u, v\rangle_{V} & =\langle\mathcal{A}(\varepsilon(u)), \varepsilon(v)\rangle_{\mathcal{H}},+j(u, v), \\
\left\langle f_{\eta}(t), v\right\rangle_{V} & =\langle f(t), v\rangle_{V}-\langle\eta(t), \varepsilon(v)\rangle_{\mathcal{H}}
\end{aligned}
$$

for all $u, v \in V, t \in[0, T]$. Let $u_{1}, u_{2} \in V$. Using (4.3) and (3.25), we find

$$
\begin{aligned}
\left\langle B u_{1}-B u_{2}, u_{1}-u_{2}\right\rangle_{V}= & \left\langle\mathcal{A}\left(\varepsilon\left(u_{1}\right)\right)-\mathcal{A}\left(\varepsilon\left(u_{2}\right)\right), \varepsilon\left(u_{1}\right)-\varepsilon\left(u_{2}\right)\right\rangle_{\mathcal{H}} \\
& +\int_{\Gamma_{3}}\left(p_{\nu}\left(u_{1 \nu}\right)-p_{\nu}\left(u_{2 \nu}\right)\right)\left(u_{1 \nu}-u_{2 \nu}\right) d a \\
& +\int_{\Gamma_{3}}\left(p_{\tau}\left(u_{1 \tau}\right)-p_{\tau}\left(u_{2 \tau}\right)\right) \cdot\left(u_{1 \tau}-u_{2 \tau}\right) d a
\end{aligned}
$$

and, keeping in mind (3.17), (3.19) and (3.20), we obtain

$$
\left\langle B u_{1}-B u_{2}, u_{1}-u_{2}\right\rangle_{V} \geq m_{\mathcal{A}}\left|u_{1}-u_{2}\right|_{V}^{2} .
$$

Using again (4.3) and (3.25) it follows that

$$
\begin{aligned}
\left\langle B u_{1}-B u_{2}, v\right\rangle_{V}= & \left\langle\mathcal{A}\left(\varepsilon\left(u_{1}\right)\right)-\mathcal{A}\left(\varepsilon\left(u_{2}\right)\right), \varepsilon(v)\right\rangle_{\mathcal{H}} \\
& +\int_{\Gamma_{3}}\left(p_{\nu}\left(u_{1 \nu}\right)-p_{\nu}\left(u_{2 \nu}\right)\right) v_{\nu} d a \\
& +\int_{\Gamma_{3}}\left(p_{\tau}\left(u_{1 \tau}\right)-p_{\tau}\left(u_{2 \tau}\right)\right) \cdot v_{\tau} d a
\end{aligned}
$$

for all $v \in V$ and, by (3.15), (3.16), we deduce that

$$
\begin{aligned}
\left|B u_{1}-B u_{2}\right|_{V} \leq & L_{\mathcal{A}}\left|u_{1}-u_{2}\right|_{V}+C_{0}\left|p_{\nu}\left(u_{1 \nu}\right)-p_{\nu}\left(u_{2 \nu}\right)\right|_{L^{2}\left(\Gamma_{3}\right)} \\
& +C_{0}\left|p_{\tau}\left(u_{1 \tau}\right)-p_{\tau}\left(u_{2 \tau}\right)\right|_{L^{2}\left(\Gamma_{3}\right)^{N}} .
\end{aligned}
$$

Inequality (4.5) shows that $B$ is a strongly monotone operator on $V$. Moreover, inequality (4.6) and assumptions (3.19), (3.20) imply that $B: V \rightarrow V$ is continuous. Therefore, using a standard result for nonlinear equations (see, e.g., [3], Corollary 15), there exists a unique element $v_{\eta}(t) \in V$ such that

$$
B v_{\eta}(t)=f_{\eta}(t) \quad \forall t \in[0, T] .
$$

Let $\sigma_{\eta}:[0, T] \rightarrow \mathcal{H}$ be defined by (4.1). Using (4.7), (4.3) and (4.4) it follows that the pair $\left\{v_{\eta}, \sigma_{\eta}\right\}$ solves (4.1) and (4.2) for all $t \in[0, T]$. Choosing $v= \pm \varphi$ where $\varphi \in \mathcal{D}$ in (4.2) yields

$$
\left\langle\sigma_{\eta}, \varepsilon(\varphi)\right\rangle_{\mathcal{H}}=\langle f(t), \varphi\rangle_{V} \quad \forall t \in[0, T]
$$

and, using (3.23), we find

$$
\operatorname{Div} \sigma_{\eta}(t)+f_{0}(t)=0 \quad \text { in } \Omega, \quad \forall t \in[0, T] .
$$

The assumption (3.21) and (4.8) imply that $\sigma_{\eta}(t) \in \mathcal{H}_{1}$ for all $t \in[0, T]$. 
Now, let $t_{1}, t_{2} \in[0, T]$. Using (4.7), (4.5) and algebraic manipulations we obtain

$$
m_{\mathcal{A}}\left|v_{\eta}\left(t_{1}\right)-v_{\eta}\left(t_{2}\right)\right|_{V} \leq\left|f\left(t_{1}\right)-f\left(t_{2}\right)\right|_{V}+\left|\eta\left(t_{1}\right)-\eta\left(t_{2}\right)\right|_{\mathcal{H}} .
$$

Moreover, (4.1), (3.17) and (3.15) yield

$$
\left|\sigma_{\eta}\left(t_{1}\right)-\sigma_{\eta}\left(t_{2}\right)\right|_{\mathcal{H}} \leq L_{\mathcal{A}}\left|v_{\eta}\left(t_{1}\right)-v_{\eta}\left(t_{2}\right)\right|_{V}+\left|\eta\left(t_{1}\right)-\eta\left(t_{2}\right)\right|_{\mathcal{H}} .
$$

Now, since $f:[0, T] \rightarrow V$ and $\eta:[0, T] \rightarrow \mathcal{H}$ are continuous functions, from (4.9) and (4.10) we deduce that $v_{\eta} \in C(0, T ; V)$ and $\sigma_{\eta} \in C(0, T ; \mathcal{H})$. Moreover, it follows from (4.8) and (3.21) that $\sigma_{\eta} \in C\left(0, T ; \mathcal{H}_{1}\right)$.

This concludes the existence part of Lemma 4.1. The uniqueness part follows from the unique solvability of the time-dependent nonlinear equation $(4.7)$.

Next, for all $\eta \in C(0, T ; \mathcal{H})$ let $u_{\eta}:[0, T] \rightarrow V$ denote the function

$$
u_{\eta}(t)=\int_{0}^{t} v_{\eta}(s) d s+u_{0} \quad \forall t \in[0, T] .
$$

We consider the operator $\Lambda: C(0, T ; \mathcal{H}) \rightarrow C(0, T ; \mathcal{H})$ defined by

$$
\Lambda \eta(t)=G\left(\varepsilon\left(u_{\eta}(t)\right)\right) \quad \forall \eta \in C(0, T ; \mathcal{H}), t \in[0, T] .
$$

We have the following result.

Lemma 4.2. The operator $\Lambda$ has a unique fixed point $\eta^{*} \in C(0, T ; \mathcal{H})$.

Proof. Let $\eta_{1}, \eta_{2} \in C(0, T ; \mathcal{H})$ and set $v_{i}=v_{\eta_{i}}, \sigma_{i}=\sigma_{\eta_{i}}$ for $i=1,2$. Using (4.1) and (4.2) we obtain

$$
\begin{gathered}
\sigma_{i}(t)=\mathcal{A}\left(\varepsilon\left(v_{i}(t)\right)\right)+\eta_{i}, \\
\left\langle\sigma_{i}(t), \varepsilon(v)\right\rangle_{\mathcal{H}}+j\left(v_{i}(t), v\right)=\langle f(t), v\rangle_{V} \quad \forall v \in V,
\end{gathered}
$$

for all $t \in[0, T], i=1,2$. Using now (4.13), (4.14) and algebraic manipulations we find

$$
\begin{aligned}
&\left\langle\mathcal{A}\left(\varepsilon\left(v_{1}(t)\right)\right)-\mathcal{A}\left(\varepsilon\left(v_{2}(t)\right)\right), \varepsilon\left(v_{1}(t)\right)-\varepsilon\left(v_{2}(t)\right)\right\rangle_{\mathcal{H}} \\
&+j\left(v_{1}(t), v_{1}(t)-v_{2}(t)\right)-j\left(v_{2}(t), v_{1}(t)-v_{2}(t)\right) \\
&=\left\langle\eta_{2}(t)-\eta_{1}(t), \varepsilon\left(v_{1}(t)\right)-\varepsilon\left(v_{2}(t)\right)\right\rangle_{\mathcal{H}}
\end{aligned}
$$

for all $t \in[0, T]$. Keeping in mind (3.17) and (3.15) we deduce that

$$
\begin{aligned}
\left\langle\mathcal{A}\left(\varepsilon\left(v_{1}(t)\right)\right)-\mathcal{A}\left(\varepsilon\left(v_{2}(t)\right)\right),\right. & \left.\varepsilon\left(v_{1}(t)\right)-\varepsilon\left(v_{2}(t)\right)\right\rangle_{\mathcal{H}} \\
& \geq m_{\mathcal{A}}\left|v_{1}(t)-v_{2}(t)\right|_{V}^{2} \quad \forall t \in[0, T]
\end{aligned}
$$

and, from (3.25), (3.19) and (3.20), we find

$$
j\left(v_{1}(t), v_{1}(t)-v_{2}(t)\right)-j\left(v_{2}(t), v_{1}(t)-v_{2}(t)\right) \geq 0 \quad \forall t \in[0, T] .
$$


It follows from (4.15)-(4.16) that

$$
\left|v_{1}(t)-v_{2}(t)\right|_{V} \leq \frac{1}{m_{\mathcal{A}}}\left|\eta_{1}(t)-\eta_{2}(t)\right|_{\mathcal{H}} \quad \forall t \in[0, T] .
$$

Therefore, from (4.12), (3.18), (4.11) and (4.18) we find

$$
\left|\Lambda \eta_{1}(t)-\Lambda \eta_{2}(t)\right|_{\mathcal{H}} \leq \frac{L_{G}}{m_{\mathcal{A}}} \int_{0}^{t}\left|\eta_{1}(t)-\eta_{2}(t)\right|_{\mathcal{H}} \quad \forall t \in[0, T] .
$$

Iterating this inequality $n$ times leads to

$$
\left|\Lambda^{n} \eta_{1}-\Lambda^{n} \eta_{2}\right|_{C(0, T ; \mathcal{H})} \leq\left(\frac{L_{G}}{m_{\mathcal{A}}}\right)^{n} \frac{T^{n}}{n !}\left|\eta_{1}-\eta_{2}\right|_{C(0, T ; \mathcal{H})},
$$

which implies that for $n$ sufficiently large a power $\Lambda^{n}$ of $\Lambda$ is a contraction on $C(0, T ; \mathcal{H})$. Thus, there exists a unique $\eta^{*} \in C(0, T ; \mathcal{H})$ such that $\Lambda^{n} \eta^{*}=\eta^{*}$ and $\eta^{*}$ is also the unique fixed point of $\Lambda$.

We now have all the ingredients to prove the theorem.

Proof of Theorem 3.1. Existence. Let $\eta^{*} \in C(0, T ; \mathcal{H})$ be the fixed point of $\Lambda$ and let $\left\{v_{\eta^{*}}, \sigma_{\eta^{*}}\right\}$ be the solution of the variational problem (4.1), (4.2) for $\eta=\eta^{*}$. We denote by $u_{\eta^{*}}$ the function given by (4.11) for $\eta=\eta^{*}$. We show that the pair $\left\{u_{\eta^{*}}, \sigma_{\eta^{*}}\right\}$ is a solution of problem $P_{V}$ which satisfies (3.31). Indeed, equality (3.28) follows from (4.11), (4.12) and (4.1) since

$$
v_{\eta^{*}}(t)=\dot{u}_{\eta^{*}}(t), \quad \eta^{*}(t)=\Lambda \eta^{*}(t)=G\left(\varepsilon\left(u_{\eta^{*}}(t)\right)\right) \quad \forall t \in[0, T] .
$$

Equality (3.29) is a direct consequence of (4.2). Finally, the initial condition (3.30) as well as the regularity (3.31) follow from Lemma 4.1 and (4.11).

Uniqueness. The uniqueness part of Theorem 3.1 results by using arguments similar to those in [12]. It is a consequence of the uniqueness of the fixed point of the operator $\Lambda$ given by (4.12).

5. Continuous dependence result. In this section we study the dependence of the solution to problem $P_{V}$ on perturbations of the contact conditions. We suppose in what follows that (3.17)-(3.22) hold and, for every $\alpha \geq 0$, let $p_{\nu}^{\alpha}, p_{\tau}^{\alpha}$ be a perturbation of $p_{\nu}, p_{\tau}$ which satisfies (3.19) and (3.20), respectively. We introduce the functional $j^{\alpha}$ that is obtained from $j$ by replacing $p_{\nu}, p_{\tau}$ with $p_{\nu}^{\alpha}, p_{\tau}^{\alpha}$. We now consider the following variational problem:

Problem $P_{V}^{\alpha}$. For $\alpha \geq 0$, find a displacement field $u^{\alpha}:[0, T] \rightarrow V$ and a stress field $\sigma^{\alpha}:[0, T] \rightarrow \mathcal{H}_{1}$ such that, for $t \in[0, T]$,

$$
\begin{gathered}
\sigma^{\alpha}(t)=\mathcal{A}\left(\varepsilon\left(\dot{u}^{\alpha}(t)\right)\right)+G\left(\varepsilon\left(u^{\alpha}(t)\right)\right), \\
\left\langle\sigma^{\alpha}(t), \varepsilon(v)\right\rangle_{\mathcal{H}}+j^{\alpha}\left(\dot{u}^{\alpha}(t), v\right)=\langle f(t), v\rangle_{V} \quad \forall v \in V, \\
u^{\alpha}(0)=u_{0} .
\end{gathered}
$$


We deduce from Theorem 3.1 that for each $\alpha \geq 0$ problem $P_{V}^{\alpha}$ has a unique solution $\left\{u^{\alpha}, \sigma^{\alpha}\right\}$ satisfying $u^{\alpha} \in C^{1}(0, T ; V), \sigma^{\alpha} \in C\left(0, T ; \mathcal{H}_{1}\right)$. Suppose now that the contact functions satisfy the following assumptions:

$$
\left\{\begin{array}{l}
\text { there exist } \beta_{\nu} \in \mathbb{R} \text { and } \varphi_{\nu}: \mathbb{R}_{+} \rightarrow \mathbb{R}_{+} \text {such that: } \\
\text { (a) }\left|p_{\nu}^{\alpha}(x, r)-p_{\nu}(x, r)\right| \leq \varphi_{\nu}(\alpha)\left(|r|+\beta_{\nu}\right) \quad \forall r \in \mathbb{R}, \text { a.e. } x \in \Gamma_{3} ; \\
\text { (b) } \lim _{\alpha \rightarrow 0} \varphi_{\nu}(\alpha)=0 ;
\end{array}\right.
$$

$$
\left\{\begin{array}{l}
\text { there exist } \beta_{\tau} \in \mathbb{R} \text { and } \varphi_{\tau}: \mathbb{R}_{+} \rightarrow \mathbb{R}_{+} \text {such that: } \\
\text { (a) }\left|p_{\tau}^{\alpha}(x, r)-p_{\tau}(x, r)\right| \leq \varphi_{\tau}(\alpha)\left(|r|+\beta_{\tau}\right) \quad \forall r \in \mathbb{R}^{N} \text {, a.e. } x \in \Gamma_{3} ; \\
\text { (b) } \lim _{\alpha \rightarrow 0} \varphi_{\tau}(\alpha)=0 .
\end{array}\right.
$$

Under these assumptions, we have the following convergence result.

TheOREM 5.1. The solution $\left\{u^{\alpha}, \sigma^{\alpha}\right\}$ of problem $P_{V}^{\alpha}$ converges uniformly to the solution $\{u, \sigma\}$ of problem $P_{V}$ :

$$
\begin{aligned}
u^{\alpha} \rightarrow u & \text { in } C^{1}(0, T ; V), \\
\sigma^{\alpha} \rightarrow \sigma & \text { in } C\left(0, T ; \mathcal{H}_{1}\right) \text { as } \alpha \rightarrow 0 .
\end{aligned}
$$

In addition to the mathematical interest in this result, it is of importance in applications, as it indicates that small inaccuracies in the contact conditions lead to small inaccuracies in the solution.

Proof of Theorem 5.1. Let $\alpha \geq 0$. To simplify the notation we shall not indicate explicitly the dependence on $t \in[0, T]$. Everywhere below $C$ will represent a positive constant which depends on the data but is independent of $\alpha$ and whose value may change from line to line.

Using (3.28), (3.29), (5.1) and (5.2), we obtain

$$
\begin{aligned}
\left\langle\mathcal{A}\left(\varepsilon\left(\dot{u}^{\alpha}\right)\right)-\mathcal{A}(\varepsilon(\dot{u})), \varepsilon(\right. & \left.\left.\dot{u}^{\alpha}\right)-\varepsilon(\dot{u})\right\rangle_{\mathcal{H}} \\
& +\left\langle G\left(\varepsilon\left(u^{\alpha}\right)\right)-G(\varepsilon(u)), \varepsilon\left(\dot{u}^{\alpha}\right)-\varepsilon(\dot{u})\right\rangle_{\mathcal{H}} \\
& +j^{\alpha}\left(\dot{u}^{\alpha}, \dot{u}^{\alpha}-\dot{u}\right)-j\left(\dot{u}, \dot{u}^{\alpha}-\dot{u}\right)=0 .
\end{aligned}
$$

Moreover, from (3.15), (3.17) and (3.18) it follows that

$$
\begin{gathered}
\left\langle\mathcal{A}\left(\varepsilon\left(\dot{u}^{\alpha}\right)\right)-\mathcal{A}(\varepsilon(\dot{u})), \varepsilon\left(\dot{u}^{\alpha}\right)-\varepsilon(\dot{u})\right\rangle_{\mathcal{H}} \geq C\left|\dot{u}^{\alpha}-\dot{u}\right|_{V}^{2}, \\
-\left\langle G\left(\varepsilon\left(u^{\alpha}\right)\right)-G(\varepsilon(u)), \varepsilon\left(\dot{u}^{\alpha}\right)-\varepsilon(\dot{u})\right\rangle_{\mathcal{H}} \leq C\left|u^{\alpha}-u\right|_{V}\left|\dot{u}^{\alpha}-\dot{u}\right|_{V} .
\end{gathered}
$$

We note that

$$
\begin{aligned}
j\left(\dot{u}, \dot{u}^{\alpha}-\dot{u}\right)-j^{\alpha}\left(\dot{u}^{\alpha}, \dot{u}^{\alpha}-\dot{u}\right)= & j\left(\dot{u}, \dot{u}^{\alpha}-\dot{u}\right)-j^{\alpha}\left(\dot{u}, \dot{u}^{\alpha}-\dot{u}\right) \\
& +j^{\alpha}\left(\dot{u}, \dot{u}^{\alpha}-\dot{u}\right)-j^{\alpha}\left(\dot{u}^{\alpha}, \dot{u}^{\alpha}-\dot{u}\right)
\end{aligned}
$$

and, using (3.19), (3.20), we find 


$$
\begin{aligned}
j\left(\dot{u}, \dot{u}^{\alpha}-\dot{u}\right)-j^{\alpha}\left(\dot{u}^{\alpha}, \dot{u}^{\alpha}-\dot{u}\right) \leq & j\left(\dot{u}, \dot{u}^{\alpha}-\dot{u}\right)-j^{\alpha}\left(\dot{u}, \dot{u}^{\alpha}-\dot{u}\right) \\
= & \int_{\Gamma_{3}}\left(p_{\nu}\left(\dot{u}_{\nu}\right)-p_{\nu}^{\alpha}\left(\dot{u}_{\nu}\right)\right)\left(\dot{u}_{\nu}^{\alpha}-\dot{u}_{\nu}\right) d a \\
& +\int_{\Gamma_{3}}\left(p_{\tau}\left(\dot{u}_{\tau}\right)-p_{\tau}^{\alpha}\left(\dot{u}_{\tau}\right)\right) \cdot\left(\dot{u}_{\tau}^{\alpha}-\dot{u}_{\tau}\right) d a .
\end{aligned}
$$

Now, in view of (5.4), (5.5) and (3.16), the previous inequality implies

$$
j\left(\dot{u}, \dot{u}^{\alpha}-\dot{u}\right)-j^{\alpha}\left(\dot{u}^{\alpha}, \dot{u}^{\alpha}-\dot{u}\right) \leq C\left(\varphi_{\nu}(\alpha)+\varphi_{\tau}(\alpha)\right)\left|\dot{u}_{\alpha}-\dot{u}\right|_{V} .
$$

Combining (5.7)-(5.10) we deduce that

$$
\left|\dot{u}^{\alpha}-\dot{u}\right|_{V} \leq C\left|u^{\alpha}-u\right|_{V}+C\left(\varphi_{\nu}(\alpha)+\varphi_{\tau}(\alpha)\right) .
$$

Now, an integration over $(0, t)$ and (3.30), (5.3) and (5.11) lead to

$$
\begin{aligned}
\left|u^{\alpha}(t)-u(t)\right|_{V} & \leq C \int_{0}^{t}\left|\dot{u}^{\alpha}(s)-\dot{u}(s)\right|_{V} d s \\
& \leq C \int_{0}^{t}\left|u^{\alpha}(s)-u(s)\right|_{V} d s+C\left(\varphi_{\nu}(\alpha)+\varphi_{\tau}(\alpha)\right)
\end{aligned}
$$

for all $t \in[0, T]$, and a Gronwall-type inequality yields

$$
\left|u^{\alpha}-u\right|_{V} \leq C\left(\varphi_{\nu}(\alpha)+\varphi_{\tau}(\alpha)\right)
$$

Moreover, from (5.1), (3.28), (3.17) and (3.18), we obtain

$$
\left|\sigma^{\alpha}-\sigma\right|_{\mathcal{H}} \leq C\left(\left|\dot{u}^{\alpha}-\dot{u}\right|_{V}+\left|u^{\alpha}-u\right|_{V}\right)
$$

and, since by (4.8), $\operatorname{Div} \sigma=\operatorname{Div} \sigma^{\alpha}=-f_{0}$, we find

$$
\left|\sigma^{\alpha}-\sigma\right|_{\mathcal{H}_{1}} \leq C\left(\left|\dot{u}^{\alpha}-\dot{u}\right|_{V}+\left|u^{\alpha}-u\right|_{V}\right)
$$

Using now (5.11)-(5.13) yields

$$
\left|\sigma^{\alpha}-\sigma\right|_{\mathcal{H}_{1}} \leq C\left(\varphi_{\nu}(\alpha)+\varphi_{\tau}(\alpha)\right) .
$$

Finally, it follows from (5.11), (5.12) and (5.14) that

$$
\left|u^{\alpha}-u\right|_{C^{1}(0, T ; V)}+\left|\sigma^{\alpha}-\sigma\right|_{C\left(0, T ; \mathcal{H}_{1}\right)} \leq C\left(\varphi_{\nu}(\alpha)+\varphi_{\tau}(\alpha)\right) .
$$

Theorem 5.1 is now a consequence of (5.4), (5.5) and (5.15).

\section{References}

[1] A. Amassad and M. Sofonea, Analysis of a quasistatic viscoplastic problem involving Tresca friction law, Discrete Cont. Dynam. Systems 4 (1998), 55-72.

[2] L.-E. Anderson, A quasistatic frictional problem with normal compliance, Nonlinear Anal. 16 (1991), 347-370.

[3] H. Brezis, Equations et inéquations non linéaires dans les espaces vectoriels en dualité, Ann. Inst. Fourier (Grenoble) 18 (1968), no. 1, 115-175. 
[4] M. Cocu, E. Pratt and M. Raous, Formulation and approximation of quasistatic frictional contact, Internat. J. Engrg. Sci. 34 (1996), 783-798.

[5] G. Duvaut et J. L. Lions, Les Inéquations en Mécanique et en Physique, Dunod, Paris, 1972.

[6] I. R. Ionescu and M. Sofonea, Functional and Numerical Methods in Viscoplasticity, Oxford Univ. Press, Oxford, 1993.

[7] N. Kikuchi and J. T. Oden, Contact Problems in Elasticity: A Study of Variational Inequalities and Finite Element Methods, SIAM, Philadelphia, 1988.

[8] A. Klarbring, A. Mikelić and M. Shillor, The rigid punch problem with friction, Internat. J. Engrg. Sci. 29 (1991), 751-768.

[9] J. Nečas and I. Hlaváček, Mathematical Theory of Elastic and Elastoplastic Bodies: an Introduction, Elsevier, Amsterdam, 1981.

[10] P. D. Panagiotopoulos, Inequality Problems in Mechanics and Applications, Birkhäuser, Basel, 1985.

[11] M. Raous, M. Jean and J. J. Moreau, Contact Mechanics, Plenum Press, New York, 1995.

[12] M. Rochdi, M. Shillor and M. Sofonea, A quasistatic viscoelastic contact problem with normal compliance and friction, J. Elasticity 51 (1998), 105-126.

[13] - - - - , A quasistatic contact problem with directional friction and damped response, Appl. Anal. 68 (1998), 409-422.

[14] - , - - - Analysis of a quasistatic viscoelastic problem with friction and damage, Adv. Math. Sci. Appl. 10 (2000), 173-189.

[15] M. Sofonea and M. Shillor, Variational analysis of quasistatic viscoplastic contact problems with friction, Comm. Appl. Anal., to appear.

Laboratoire de Théorie des Systèmes Université de Perpignan 52 Avenue de Villeneuve 66860 Perpignan Cedex, France

E-mail: awbi@univ-perp.fr sofonea@univ-perp.fr
Laboratoire de Mathématiques Appliquées CNRS UMR 6620 Université Blaise Pascal (Clermont-Ferrand II) 63177 Aubière Cedex, France E-mail: essoufi@ucfma.univ-bpclermont.fr 